\title{
Schedule Of Assessments
}

National Cancer Institute

\section{Source}

National Cancer Institute. Schedule Of Assessments. NCI Thesaurus. Code C142678.

A sequential representation of planned protocol events and activities that pertain to patient assessment within the study. 